\title{
Additive manufacturing and foundry innovation
}

\author{
*Yu-sheng Shi" ${ }^{1,2}$ Jin-liang Zhang ${ }^{1,2}$, Shi-feng Wen ${ }^{1,2}$, Bo Song ${ }^{1,2}$, Chun-ze Yan ${ }^{1,2}$, Qing-song Wei", Jia-min Wu", \\ Ya-jun Yin', Jian-xin Zhou', Rui Chen ${ }^{3}$, Wei Zhou ${ }^{4}$, He-ping Jia ${ }^{5}$, Huan-qing Yang ${ }^{6}$, Hai Nan ${ }^{7}$ \\ 1. State Key Laboratory of Materials Processing and Die \& Mould Technology, School of Materials Science and Engineering, Huazhong \\ University of Science and Technology, Wuhan 430074, China \\ 2. Engineering Research Center of Ceramic Materials for Additive Manufacturing, Ministry of Education, Wuhan 430074, China \\ 3. Shenyang Research Institute of Foundry Co., Ltd., Shenyang 110011, China \\ 4. Liaoning Senyuan Additive Manufacturing Technology Co., Ltd., Anshan 114000, Liaoning, China \\ 5. Jiangsu Jiuyu Machinery Co., Ltd., Suzhou 215300, China \\ 6. Xi'an Space Engine Company Co., Ltd., Xi'an 710100, China \\ 7. Beijing Institute of Aeronautical Materials, Beijing 100095, China
}

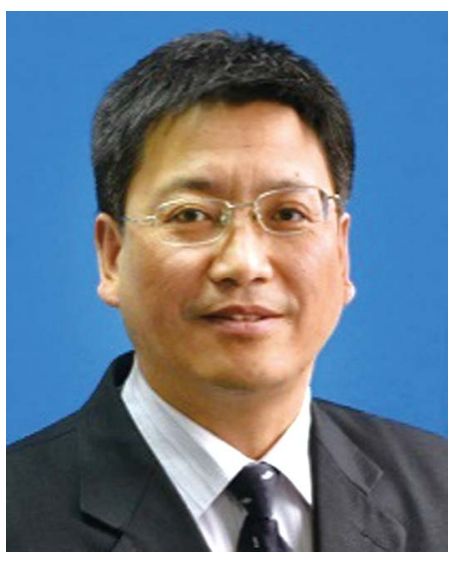

*Yu-sheng Shi

Male, born in 1962, Ph.D., Scholar Leading Distinguished Professor of Huazhong University of Science and Technology. He is currently the Vice Director Member of the Special Materials Group of the National Additive Manufacturing Standardization Technical Committee, the Director of the Expert Committee of the Additive Manufacturing Process Technology Center of China Aerospace Science and Technology Corporation, and the Vice Director Member of the Additive Manufacturing Branch of the Chinese Mechanical Engineering Society. He has won the award of the China's Top Ten Scientific and Technological Advancements, one Second Prize of National Technological Invention, and two Second Prizes of National Science and Technological Progress. He has won the Special Prize of China Invention and Entrepreneurship Award and Contemporary Inventor, Top Ten Outstanding Innovators of Chinese Science, Top Ten National Outstanding Scientific and Technological Worker Nomination Award. His team was selected as the Innovation Team of Hubei Province and the Ministry of Education.

E-mail: shiyusheng@hust.edu.cn

Received: 2021-05-10

Accepted: 2021-07-05
Abstract: Additive manufacturing is expected to transform and upgrade the traditional foundry industry to realize the integrated manufacturing and rapid and low-cost development of high-performance components with complex shapes. The additive manufacturing technology commonly applied in casting mold preparation (fusible molds, sand molds/cores and ceramic cores) mainly includes selective laser sintering (SLS) and binder injection threedimensional printing (3DP). In this work, the research status of SLS/3DPcasting processes on material preparation, equipment development, process optimization, simulation and application cases in aerospace, automotive and other fields were elaborated. Finally, the developing trends of the additive manufacturing technology in the future of foundry field are introduced, including multi-material sand molds (metal core included), ceramic core-shell integration and die-casting dies with conformal cooling runners.

Key words: additive manufacturing; foundry; selective laser sintering; 3D printing; multiple materials; die-casting dies

CLC numbers: TG221; Document code: A;

Article ID: 1672-6421(2021)04-286-10

\section{Introduction}

As a basic industry in the global economy construction, the foundry industry plays an important role in the prosperity of the world economy. With growing lightweight and functional requirements in high-end equipment, high-performance large-scale casting components are becoming more and more complex. For example, compared with the aero engine intermediary receiver in the 2010 s, the new generation intermediary receiver adopts a more complicated curved gooseneck-shaped runner and blind cavity structure, which reduces the weight by about $30 \%$, increasing the thrust-to-weight ratio by about $20 \%$, and reducing fuel consumption by about $25 \%$ (Fig. 1). At present, traditional casting technology is one of the main forming technologies for the key metal components of high-end equipment. The process flow is roughly divided into three stages: split mold (core) design, mold manufacturing and mold casting. Although the castings formed by the above-mentioned traditional processes have stable process and good performance, and also have formed a fixed production line, they still have the problems of long split casting and splicing process and too 

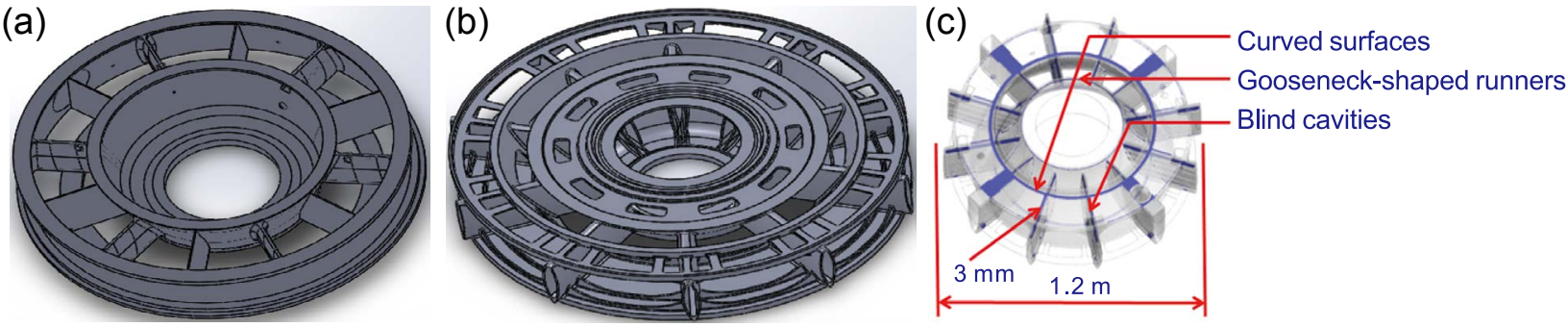

Fig. 1: Intermediary receiver in 2010 s (a), new generation intermediary receiver (b), and structural features of new generation intermediary receiver (c)

many influencing factors, which make it difficult to realize the integrated forming of complex components. Therefore, it is deemed urgent to develop an advanced casting technology which can realize the integrated forming of high-performance complex components. The integrated casting technology of large complex components embodies the manufacturing level of a country, where the international competition is fierce. The US Additive Manufacturing Roadmap, EU Horizon 2020 and China Foundry Technology Roadmap all have put forward the development ideas and goals of advanced integrated casting technology.

Additive manufacturing (AM) is a new type of forming method where materials are stacked layer by layer. It is driven by the three-dimensional data of the part, which is sliced and layered. In this way, complex three-dimensional forming is transformed into a simple two-dimensional forming process from the bottom up ${ }^{[1-3]}$. Compared with traditional equivalent material manufacturing (casting, forging and welding, et al) and subtractive manufacturing (lathe turning, milling and grinding, et al) technologies, AM greatly reduces the difficulty of forming complex components without the need of molds, tools or fixtures. Additive manufacturing is expected to solve the technical problem of overall manufacturing of high-performance metallic components with complex shapes, which is difficult to complete with traditional methods. Additive manufacturing is listed as an advanced manufacturing technology that is regarded to improve national competitiveness and meet future challenges by strategic plans such as "America Makes" of the United States, "Horizon 2020" of the European Union, "Industry 4.0" of Germany and "Made in China 2025". Combining additive manufacturing with traditional casting technology, that is, using additive manufacturing technology to prepare complex molds and then casting with them, is expected to realize the rapid overall manufacturing of large, complex and special-shaped key metal components in the aerospace, military and other fields. Furthermore, additive manufacturing-casting manufacturing technology can realize the rapid and low-cost development of new products, and improve the innovation ability and level of related fields. The advantages of additive manufacturingcasting technology can be summarized as follows: (1) Green design: combined with additive manufacturing, the complexstructured parts can be designed and formed so that the casting products can realize light weight and reduce materials consumption; (2) Green manufacturing: preparing molds using additive manufacturing technology to replace machining methods can reduce material loss and energy consumption and improve production efficiency. Additionally, repairing technology by additive manufacturing can reduce the scrap rate of high-end castings and realize green manufacturing; (3) Performance improvement: additive manufacturing technology can realize the overall design and casting of complex structural components, which is beneficial to the improvement of component performance; (4) Short production time and low cost: additive manufacturing-casting technology can improve the development speed of new casting products and reduce the research cost and risk of the product preparation, which can meet the single and small-batch casting demand.

\section{Additive manufacturing technology}

At present, the additive manufacturing technology commonly used in mold preparation mainly includes selective laser sintering (SLS) and binder injection three-dimensional printing (3DP).

\subsection{SLS technology}

The processing principle of SLS is as follows: The laser beam scans the powder bed selectively according to the section information of the three-dimensional model to sinter the powder material and bond them together. After a layer is finished, the working platform drops by a layer thickness, and repeat this step until the whole mold processing is completed ${ }^{[4,5]}$. The main characteristics of this technology are: (1) High material utilization rate; (2) High strength and stable performance of SLS-fabricated mold. SLS is mostly

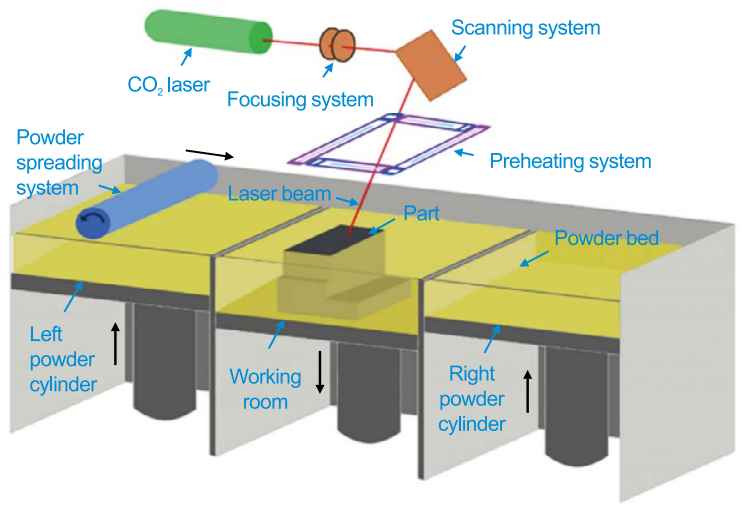

Fig. 2: Schematic diagram of SLS 
used in engineering materials with excellent performance; (3) Wide variety of forming materials: SLS is suitable for the preparation of polymers, ceramics and composite materials ${ }^{[6]}$.

The main difficulty of SLS-casting technology is that the mold (core) material is difficult to prepare, that is, it is difficult to prepare the powder material that meets the requirements of both SLS and casting processes. Increasing the mole fraction of a benzene ring and the binder content of ceramics and sand molds (cores) is beneficial to increase the strength of the molds (cores), but it will result in more defects or even scrapping of the castings. Furthermore, the strength of the molds (cores) will decrease, which cannot meet the requirements of casting. Therefore, the influence of material properties on the strength of the mold (core) is one of the research hotspots in this field. The research team of Professor Shi in Huazhong University of Science and Technology proposed an effective method for the preparation of high-performance laser sintering materials for different core materials. For fusible mold materials, the block modification method was used to reduce the mole fraction of a benzene ring and increase the rigidity of molecular chains. For ceramic core materials, the interface organic modification method was used to improve the bonding force between the adhesive and the matrix. For sand (core) materials, the active group modification method was adopted to effectively improve the reactivity of the materials and make it fully cured. Additionally, a distillation cooling method for uniform cooling control was proposed, and the facilities such as program control high-pressure reactor and distillation cooling solvent recovery device were developed. The above research achievement broke through the key technology of large-scale powder production and realized the preparation of the powder materials that satisfied both SLS and casting processes. The developed spherical or nearly spherical powder has a particle size of 100-150 $\mu \mathrm{m}$, ash content $<0.3 \%$ and gas release $<18.6 \mathrm{~g} \cdot \mathrm{mL}^{-1}$ (Fig. 3 ).
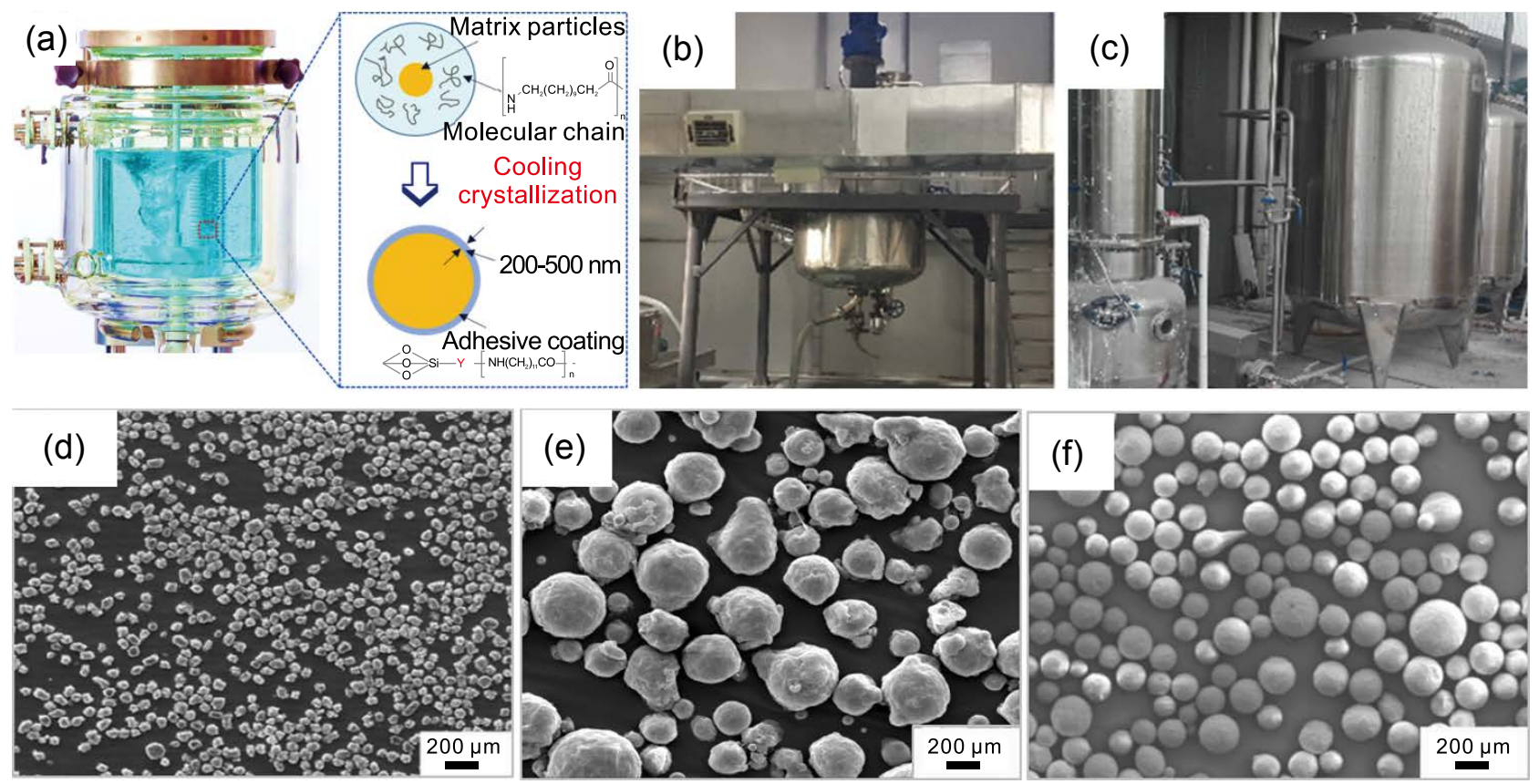

Fig. 3: Distillation cooling uniform cooling control method and SLS powder: (a) schematic diagram, (b) programcontrolled high-pressure reactor, (c) distillation cooling solvent recovery device, (d-f) SLS powder for fusible molds, ceramic and sand molds, respectively

In addition, relying on the technology transformation platform (Wuhan Huake 3D Technology Co., Ltd.), many kinds of SLS additive manufacturing facilities were independently designed and developed, such as fusible molds, ceramic cores and sand mold (core) additive manufacturing equipment (Fig. 4). To realize the four-laser coordinated scanning and high-precision stitching, a controllable random disturbance curve dynamic process design method was proposed, which could realize the real-time automatic generation of the split path between the four-laser scanning area, and solve the problem of poor connection strength at the boundary of the laser scanning area when large-size fusible molds and sand molds are integrally formed [Fig. 5(a)]. A method based on the combination of orderly pattern segmentation and scanning commutation was proposed to achieve the uniformity of the strength and the reduction of internal stress during the forming process of largescale sand molds, which solves the problem of deformation and low precision of the molds [Fig. 5(b)]. On this basis, a series of SLS equipment has been developed. Among them, the four-laser and four-galvanometer SLS equipment named HK C17030 has the world's largest working platform, and the forming size could reach $1,700 \times 1,700 \times 600 \mathrm{~mm}^{3[7]}$. To obtain the overall optimal casting process of SLS-fabricated large complex molds, Jiang et al. ${ }^{[8]}$ put forward a process optimization method and defect formation mechanism, and established an octree grid data structure, realizing the selfadaptive grid division of complex geometric entities [Fig. 5(c)]. This technology effectively solved the difficult problems of 


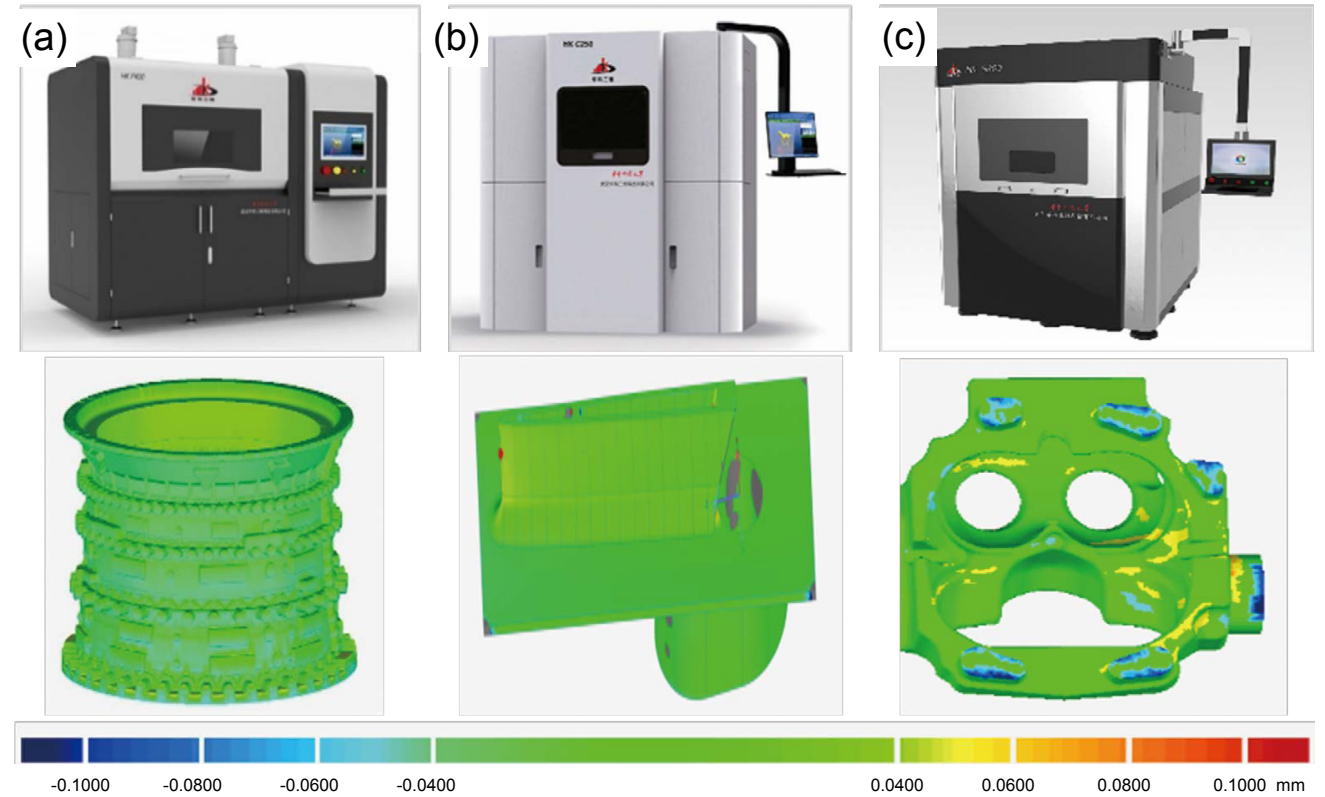

Fig. 4: SLS equipment and products with high accuracy: (a) fusible mold; (b) ceramic cores; (c) sand mold

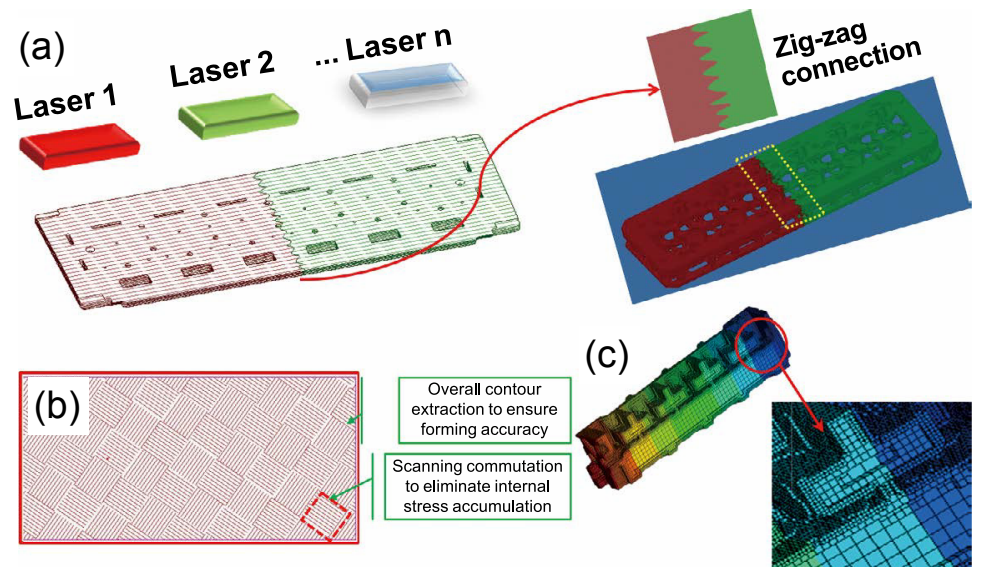

Fig. 5: SLS process optimization and simulation methods: (a) controllable random disturbance curve dynamic process design method; (b) graphic orderly segmentation and commutation scanning method; (c) octree grid data structure

discretization and low calculation efficiency of the complex interface calculation area. The calculation efficiency was improved more than threefold.

Based on the above mentioned SLS-casting technologies, the size of the SLS-fabricated mold approaches to $1.4 \mathrm{~m} \times 1.4 \mathrm{~m}$, the fusible mold strength is close to $7.54 \mathrm{MPa}$, the sand mold strength is $6.9 \mathrm{MPa}$, the forming efficiency is $4,000 \mathrm{~cm}^{3} \cdot \mathrm{h}^{-1}$, and the accuracy is $0.2 \mathrm{~mm}$.

The simulation of complex castings based on SLS process was accomplished. Through the process optimization, casting defects were reduced by more than $30 \%$. The above innovations have been applied to a variety of key large-scale components, for example the EMUSIC project. In the field of investment casting, typical application cases are titanium alloy intermediate casings, as well as high-strength stainless steel turbo pump casings and centrifugal wheels. A titanium alloy intermediate casing is the main load-bearing component of a new generation of aeroengines. When using the manufacturing methods where forming and assembly processes are divided, the forming accuracy is poor and metallurgical defects are obviously generated which cannot meet the assembly requirements, and therefore, the development of new aero-engines is severely restricted. When SLS-casting integral forming technology is utilized [Fig. 6(a)] (that is, using SLS technique to fabricate mold (core) and using numerical simulation method to optimize the casting process), hole defects are reduced by $20 \%$, casting accuracy is increased from CT9 to CT7-CT6, manufacturing efficiency is increased by more than 6 times, and weight is reduced by $30 \%$.

The turbo pump is called the heart of the space engine. The service environment of the turbo pump casings and centrifugal wheels of the new generation of main-power aerospace liquid oxygen kerosene engine is extremely harsh. The shell (static part) is used as a high-pressure bearing part and works in an environment of strong vibration and severe erosion. The working pressure can reach up to $65 \mathrm{MPa}$. The high-speed rotating centrifugal wheel (dynamic part) works in a liquid oxygen environment at $-196{ }^{\circ} \mathrm{C}$ with the rotating speed of over $27,000 \mathrm{r} \cdot \mathrm{min}^{-1}$. It is difficult to meet the accuracy and performance 
(a)
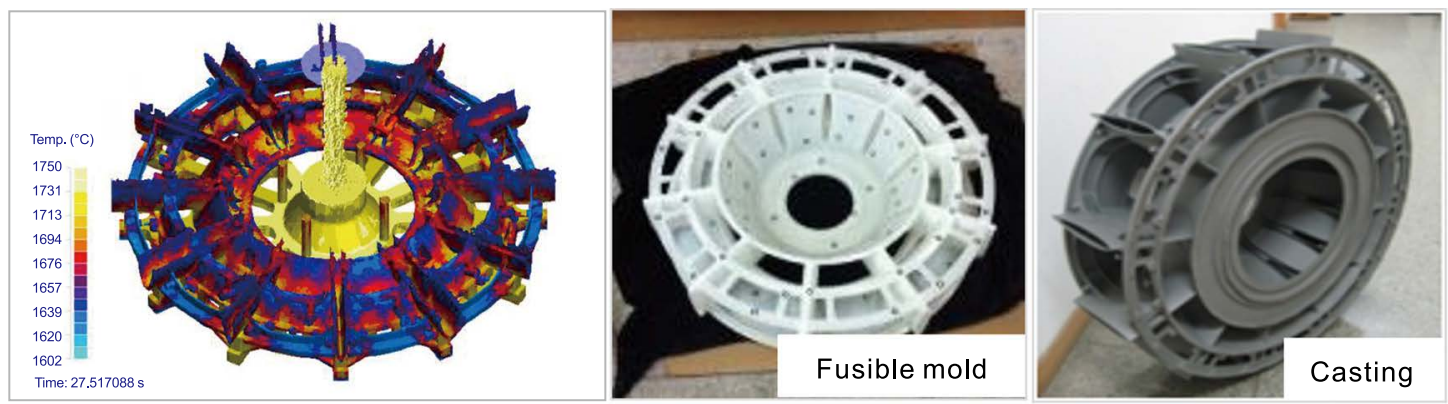

(b)
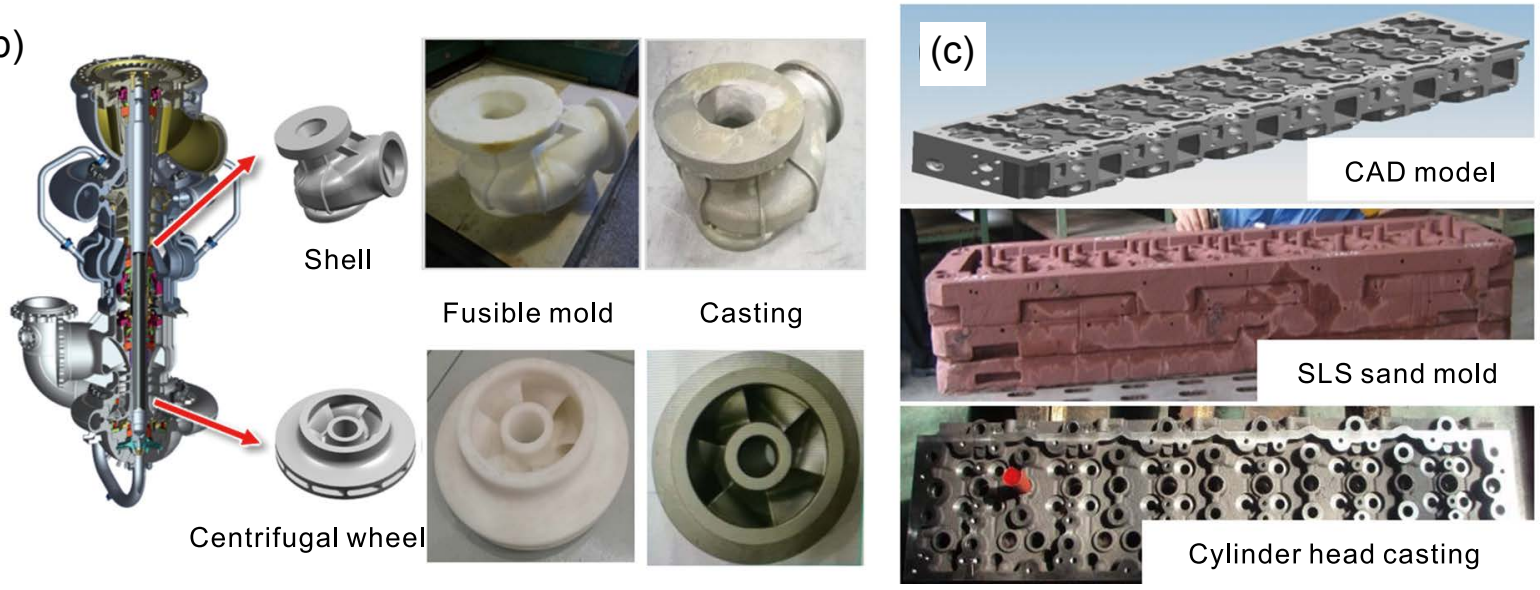

Fig. 6: SLS-casting application cases: (a) titanium alloy intermediate casing; (b) high-strength stainless steel turbo pump casing and centrifugal wheel; (c) vermicular iron six-cylinder diesel engine cylinder head sand mold

requirements of new generation rocket engines using traditional manufacturing methods. Using SLS-casting integral forming technology, the complex mold of the turbo pump (core) is directly fabricated by SLS technique without the mold [Fig. 6(b)]. The high-performance complex Al-Mg alloy turbo pump is then manufactured by an integral casting process, hot isostatic pressing and numerical optimization system. The hole defects are reduced by $30 \%$, the manufacturing accuracy is increased from CT 8 to CT6-CT5, the turbo pump head is increased by $2.9 \%$, and the efficiency is increased by $1.2 \%$. These improvements have made outstanding contributions to major national projects such as the batch production of the latest CZ5/6/7 type III engine, the 480 theavy-duty carrier fire detection engine, and the deep variable thrust lunar engine. Another application case of SLS-casting integral forming technology is the cylinder head of the vermicular iron six-cylinder diesel engine, which is one of the most complex components in the automobile engine [Fig. 6(c)]. The maximum dimension of the component is more than $1 \mathrm{~m}$, and the thinnest wall thickness is $5 \mathrm{~mm}$. When using SLS-casting integral forming technology, the working hours of mold manufacturing and repeated modification can be saved. At the same time, the number of trial productions is greatly reduced by numerical simulation technology. The casting cycle is shortened from 5-6 months to less than 20 days.

\subsection{Three-dimensional printing technology}

Three-dimensional printing (3DP) is also called binder injection technology. The technical principle of binder injection 3DP is shown in Fig. 7. The nozzle selectively

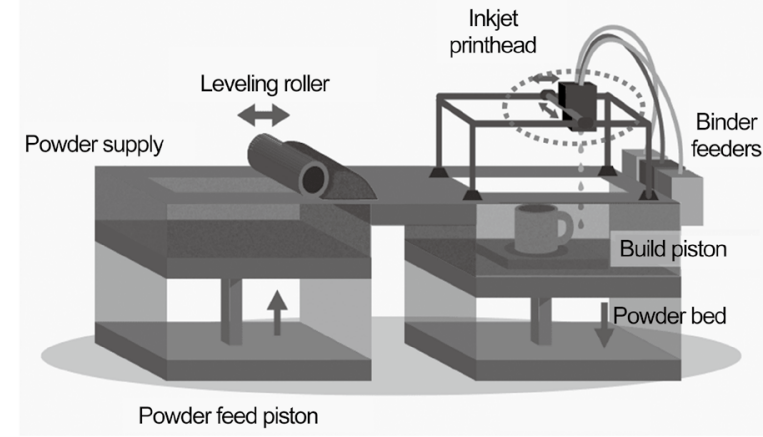

Fig. 7: Schematic diagram of 3DP

ejects micro-droplets on the surface of the thin powder layer according to the contour information of each layer of the threedimensional model. Then, the binder and the powder undergo a physical and chemical reaction and produce a certain binding force. After that, the cross-sectional profile of the current layer is formed. The layers are fixed by the bonding effect of the droplets until the 3D model is printed, and the bonded part is post-processed to form a $3 \mathrm{D}$ solid model or part ${ }^{[9,10]}$. 3DP technology uses hundreds or even tens of thousands of nozzles to spray, forming a part. Therefore, the forming size is large, and the efficiency is significantly higher than the technologies that use a high-energy beam such as laser. There is no obvious stress inside the component, and it also has advantages in the production cost. At present, 3DP technology has been successfully applied to the printing of sand molds and plastic parts, and has begun to expand to metal printing in the United States, Germany and other countries ${ }^{[11-13]}$. 
In the selection of 3DP powders, factors such as mold strength, nozzle stability, gas releasing and casting temperature ${ }^{[14]}$ need to be considered. Powder bonding methods mainly include hydration method, metal salt method, organic binder method, inorganic binder method and solvent method ${ }^{[11]}$. The applicable forming materials are shown in Table 1. The powder bonding process can be roughly divided into the following three steps: (1) The nozzle moves to selectively deposit the adhesive on the surface of the powder layer, and the adhesive penetrates the powder layer through capillary action and dissolves the resin on the surface of the coated sand. (2) After the adhesive volatilizes, the dissolved resin separates out, and the particles are bonded together through the bonding bridge to form an initial blank. (3) After a low-temperature sintering of the initial blank, the resin and the adhesive undergo cross-linking reaction to obtain a sand mold (core) for casting.

At present, many companies or research institutions at home and abroad have carried out research and development of 3DP

Table 1: Commonly used powder bonding methods

$\begin{array}{ccc}\text { Bonding methods } & \text { Forming materials } & \text { Residual } \\ \text { Hydration } & \text { Plaster or cement } & \text { No } \\ \text { Metal salt } & \text { Metal and metal salt } & \text { No } \\ \text { Organic binder } & \text { All the materials } & \text { Uncertain } \\ \text { Inorganic binder } & \text { All the materials } & \text { Yes } \\ \text { Solvent } & \text { Polymer Materials } & \text { Not sure }\end{array}$

equipment (Table 2). The VX4000 equipment developed by German Voxeljet company has a working cylinder size of $4 \mathrm{~m}$ $\times 2 \mathrm{~m} \times 1 \mathrm{~m}$, a nozzle width of $1,120 \mathrm{~mm}$, a printing accuracy of about $\pm 0.2 \mathrm{~mm}$, and a forming speed of $15.4 \mathrm{~mm} \cdot \mathrm{h}^{-1}{ }^{[15]}$. The raw material of the forming powder is sodium silicate particles and silica sand material. The water-based binder can dissolve the sodium silicate in the powder, and it can be physically dehydrated and hardened after heating. Its advantages are environmental protection and low gas generation. Silica sand is difficult to be mechanically mixed uniformly, the mold strength is low, and the bending strength is only $2.2-2.8 \mathrm{MPa}^{[14]}$. The working cylinder size of the Exerial equipment developed by ExOne in the United States is $2.2 \mathrm{~m} \times 1.2 \mathrm{~m} \times 0.7 \mathrm{~m}$, the printing layer thickness is $0.28-0.5 \mathrm{~mm}$, the printing accuracy is $\pm 0.1 \mathrm{~mm}$, and the forming speed is $300-400 \mathrm{~L} \cdot \mathrm{h}^{-1}{ }^{[16]}$. The forming raw materials are raw sand and curing agent, and the self-hardening furan resin is used as a binder to react with the curing agent to cure and form the sand mold. The working cylinder of the PCM1200 equipment developed by Guangdong Fenghua Zhuoli Company has a size of $1.2 \mathrm{~m} \times 1 \mathrm{~m} \times 0.65 \mathrm{~m}$, the printing layer thickness is $0.2-0.5 \mathrm{~mm}$, the sand mold dimensional accuracy is $\pm 0.3 \mathrm{~mm}$, and the building speed is $400-600 \mathrm{~mm} \cdot \mathrm{s}^{-1}{ }^{[17]}$. Using silica sand as the molding material, furan resin as the binder, and toluene sulfonic acid as the catalyst, the printed mold has high strength and no post-processing is required, but the required amount of resin and gas is great. The 3DP printing equipment developed by Ningxia Kocel Group has a height of $5.2 \mathrm{~m}$, a weight of $35 \mathrm{t}$, and a printing accuracy of $\pm 0.3 \mathrm{~mm}^{[18]}$.

Table 2: Representative 3DP equipment abroad

\begin{tabular}{|c|c|c|c|c|c|}
\hline Company & Composition & Binder & Curing method & Advantage & Disadvantage \\
\hline Voxeljet ${ }^{[15]}$ & $\begin{array}{l}\text { Raw sand and } \\
\text { sodium silicate }\end{array}$ & Water-based binder & Microwave curing & Low gas evolution & Low intensity \\
\hline ExOne $^{[16]}$ & $\begin{array}{c}\text { Raw sand and curing } \\
\text { agent }\end{array}$ & $\begin{array}{l}\text { Self-hardening furan } \\
\text { resin }\end{array}$ & $\begin{array}{l}\text { Resin reacts with } \\
\text { curing agent to cure }\end{array}$ & $\begin{array}{l}\text { No need for post } \\
\text { curing }\end{array}$ & $\begin{array}{l}\text { Complex nozzle } \\
\text { maintenance }\end{array}$ \\
\hline 3D System ${ }^{[19]}$ & $\begin{array}{c}\text { Foundry sand, } \\
\text { plaster, and additives }\end{array}$ & Vinyl resin & Reactive curing & - & $\begin{array}{l}\text { Only suitable for low } \\
\text { melting point alloys }\end{array}$ \\
\hline Soligen Technology & $\begin{array}{l}\text { Ceramic-based } \\
\text { powder }\end{array}$ & Silica sol & $\begin{array}{c}\text { Water molecules } \\
\text { evaporate and solidify }\end{array}$ & - & $\begin{array}{l}\text { Not suitable for } \\
\text { superalloys }\end{array}$ \\
\hline $\mathrm{PCM}^{[17]}$ & Raw sand & $\begin{array}{l}\text { Furan resin, toluene } \\
\text { sulfonic acid }\end{array}$ & $\begin{array}{l}\text { Reactive curing of } \\
\text { resin and catalyst by } \\
\text { dual nozzles }\end{array}$ & $\begin{array}{l}\text { High mold strength, } \\
\text { no need for special } \\
\text { post-treatment }\end{array}$ & $\begin{array}{c}\text { Large air volume, } \\
\text { low machining } \\
\text { accuracy and surface } \\
\text { quality }\end{array}$ \\
\hline
\end{tabular}

The 3DP printing equipment independently developed by authors is shown in Fig. 8(a). The maximum forming space is $2,000 \mathrm{~mm} \times 1,000 \mathrm{~mm} \times 600 \mathrm{~mm}$, which can meet the majority of the size requirements of sand casting. The 3DP printing equipment has high forming speed and efficiency, as it only takes $0-50 \mathrm{~s}$ to finish one layer. In addition, a precise inkjet system is utilized in this equipment, which can fully guarantee the long-term service life of the nozzle without clogging in the fast jet printing process. The equipment with our selfdeveloped system software and control unit can fabricate molds of coated sand, ceramics, plastics, gypsum, and their composite powder materials. 

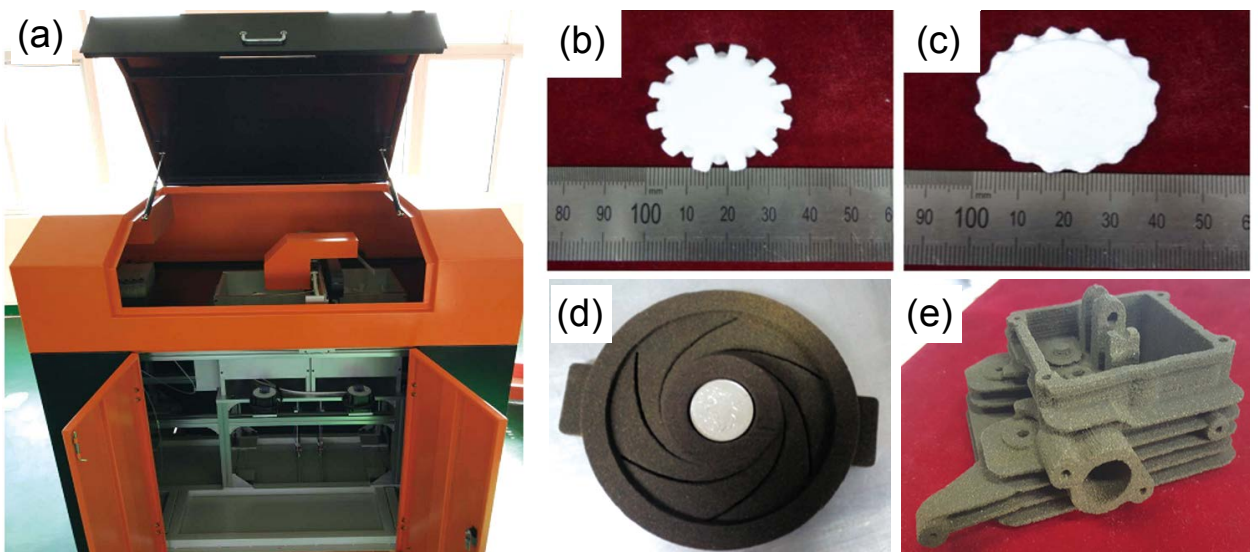

Fig. 8: Independently developed 3DP equipment (a) and molds: (b-c) fusible mold, (d-e) sand mold (core)

\section{Future developing trend of AM casting technology}

Additive manufacturing-casting integrated forming technology is expected to realize rapid integrated manufacturing of large, complex, and special-shaped key components. It can also realize the rapid and low-cost development of new products and improve the innovation ability or level of related fields.

\subsection{Multi-material additive manufacturing for sand mold with metal core}

Although the above-mentioned SLS and 3DP additive manufacturing technologies solve the manufacturing problem of complex-shaped sand mold (core), the performance requirements for the complex sand mold (core) are diversified in the casting process. Firstly, different structures and different wall thicknesses have different requirements for the refractoriness, heat storage capacity, and cooling rate of the sand mold. Therefore, individual requirements are put forward for different types of sand mold materials. Secondly, the sand mold must have a reasonable intensity gradient distribution. It must be able to meet the requirements of large size, thin wall, and slender core at room temperature. Besides, it must be able to withstand the static pressure of the molten metal, the gravity of the casting after solidification, and the pressure or tension caused by the shrinkage of the casting during the casting process. For example, manual molding mostly uses core bone and face/back sand to meet the diversified requirements of sand mold (core) performance ${ }^{[21]}$. At present, sand molds (core) fabricated by SLS and 3DP technology are often made of a single material. There are many casting defects caused by large amount of gas, such as gas hole, so it is difficult to meet the diversified requirements of sand mold (core) performance ${ }^{[22,23]}$. For this reason, Shi's team from Huazhong University of Science and Technology and Chen's team from Shenyang Research Institute of Foundry Co., Ltd. proposed the principle and method of integrated laser 3D printing of metal core bonevariable area performance complex sand mold (Fig. 9). On the basis of in-depth study of various powder feeding mechanisms, the technology and device for precise suction and feeding

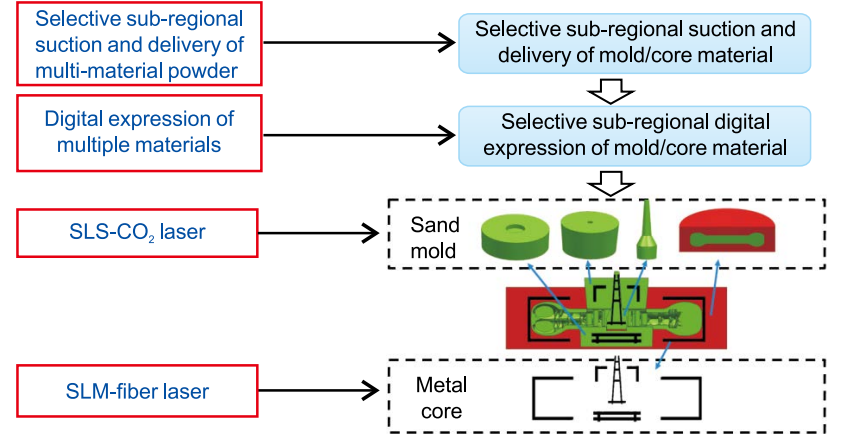

Fig. 9: Research route of laser 3D printing technology of complex metal core bone-sand mold/core with variable regional performance

of metal/non-metal multi-material powder by tiny-tubes were proposed. Multi-material integrated laser 3D printing equipment was developed, achieving the preparation of the metal core (bone) that meets the diversified requirements of casting-complex sand molds with variable performance in different areas. The detailed route is that the multi-material powder feeding system is the core component of the multimaterial laser 3D printing technology. The powder feeding system should meet the selective sub-regional suction and delivery of the molding sand/core and core material. The printing equipment software should be able to meet the digital expression of the sand/core and core material sub-regions. In addition, different lasers are used to achieve selective area forming. For example, $\mathrm{CO}_{2}$ lasers are used to form sand molds and sand core materials, and fiber lasers are used to form metal core materials.

\subsection{Integrated additive manufacturing of ceramic core and shell}

To enable the turbine blades to sustain higher intake temperatures, the design and manufacture of high-efficiency cooling air ducts inside the turbine blades are a common choice for the current advanced turbine blades. This requires the participation of ceramic cores in the blade precision casting process. During the directional solidification process, the blade ceramic core needs to be immersed in liquid metal 
above $1,500{ }^{\circ} \mathrm{C}$ for more than $90 \mathrm{~min}{ }^{[24]}$. In the subsequent solidification process, the ceramic core near the mushy zone (solid-liquid coexistence area) locally needs to withstand a temperature gradient of $30-80 \mathrm{~K} \cdot \mathrm{cm}^{-1}$ or even higher (in liquid metal cooling technology, the temperature gradient can be as much as $300 \mathrm{~K} \cdot \mathrm{cm}^{-1}$ higher). This process is prone to stress and cracks. These technological difficulties and complicated cooling air runner design promote strict dimensional accuracy and service performance requirements for the production and manufacture of ceramic cores. Correspondingly, a ceramic mold shell that can maintain a certain strength and stability at a high temperature of up to $1,500{ }^{\circ} \mathrm{C}$, and can ensure no cracking and softening under the large stress caused by a high temperature gradient is also the key to the formation of turbine blades. The current precision casting process for hollow blades is to prepare the ceramic core first, then wrap it in a wax mold by pressing wax, then coat the wax mold several times, hang ceramic slurry, and finally dewax and sinter to form a mold shell containing a ceramic core ${ }^{[25]}$. This process is such a complicated process that it is prone to quality problems. To solve this problem, based on additive manufacturing technology, researchers have carried out research on integrated core and shell manufacturing technology in recent years. However, the current methods are all single ceramic material $3 \mathrm{D}$ printing for integrated production. In fact, the working conditions of the core and the shell are different, and their materials and properties are required to be different. Therefore, it is necessary to develop a bi-material ceramic core/mold integrated manufacturing technology. Shi's research team proposed a directional column/single crystal turbine blade core/mold shell preparation technology, and bi-material ceramic core/mold shell integrated additive manufacturing and removal technology. Firstly, plans are made to break through the key technology of precise paving and feeding of two materials, and acquire the precise control of powder feeding, powder absorption and additive manufacturing process of two kinds of ceramic powders. For example, the nozzle ultrasonic vibration is used to feed powder, the suction nozzle negative pressure can be controlled to absorb the powder [Fig. 10(a)], and the high-speed camera is used to monitor the printing status. After realizing the precise powder absorption and feeding in different regions, research should also be carried out on the high-strength bonding mechanism of different material interfaces, the optimization of multi-material forming processes, and the influence of processes on the bonding strength of heterogeneous materials. Secondly, the additive/ finishing composite manufacturing technology barrier was broken. That is the bi-material additive manufacturing, it can also achieve close coordination with the spray finishing process to achieve precise control of the spraying process in small areas, and ultimately improve the ceramic core and the surface quality of the mold shell. Based on the above two key technological breakthroughs, the SLS additive/finishing multimaterial additive manufacturing equipment were successfully developed [Fig. 10 (b)].
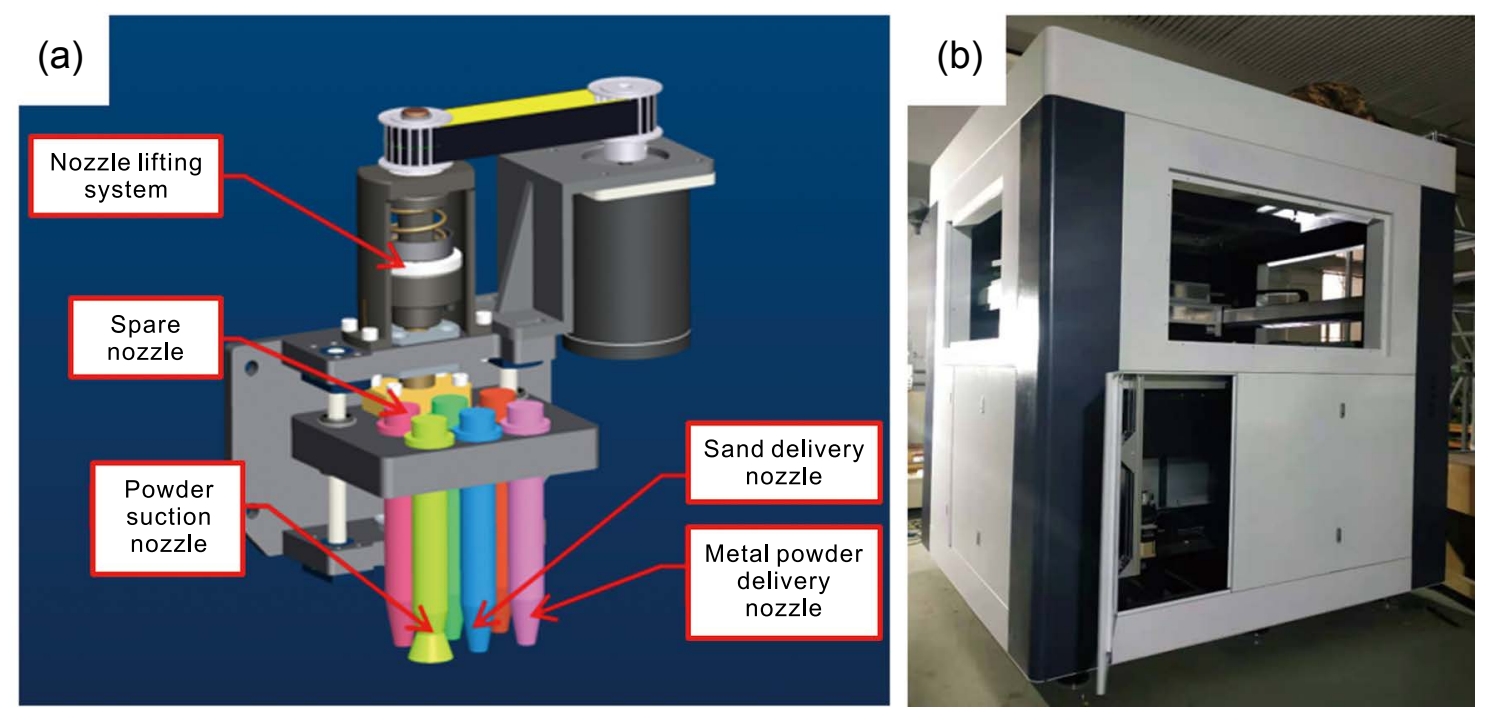

Fig. 10: Multi-material tiny-tube precise suction and powder feeding (a); SLS additive/finishing multi-material additive manufacturing equipment (b)

\subsection{Additive/subtractive manufacturing for die-casting die with conformal cooling runners}

Compared with the traditional cooling method, the mold conformal cooling method can achieve uniform cooling, significantly improving the quality of the product and reducing the cooling time ${ }^{[26]}$. However, it is difficult to integrally form complex structural components with internal conformal cooling channels by traditional processing methods such as equivalent and subtractive materials, especially materials with a high forming temperature such as stainless steel. Zhou et al. ${ }^{[27]}$ developed a multi-metal powder core-wire material synergistic arc additive/subtractive technology and equipment. The integrated process of preparation and forming 
of wire 3D printing solves the overall forming problems of hot working dies for conformal cooling channels. Compared with common metal additive manufacturing technologies, the arc fuse additive manufacturing technology (Fig. 11) has the following advantages: non-requirement of vacuum or inert gas environment, high energy utilization rate $(90 \%)$, large forming size, low cost, high forming efficiency $\left(>20 \mathrm{~kg} \cdot \mathrm{h}^{-1}\right)$, and easy forming of multi-material. The maximum forming space of the developed additive/subtractive manufacturing equipment is $3.0 \times 2.0 \times 1.5 \mathrm{~m}^{3}$ [Fig. 12(a)], with the dimensional accuracy of $\pm 10 \mu \mathrm{m}$, the part density of $>98 \%$, and the surface roughness of $\mathrm{Ra}=1.6 \mu \mathrm{m}$. The mold service temperature is raised from $700{ }^{\circ} \mathrm{C}$ to $1,600^{\circ} \mathrm{C}$. Compared with the traditional hot working mold, the service life is increased by 4 times, with a cost saving of $30 \%$.
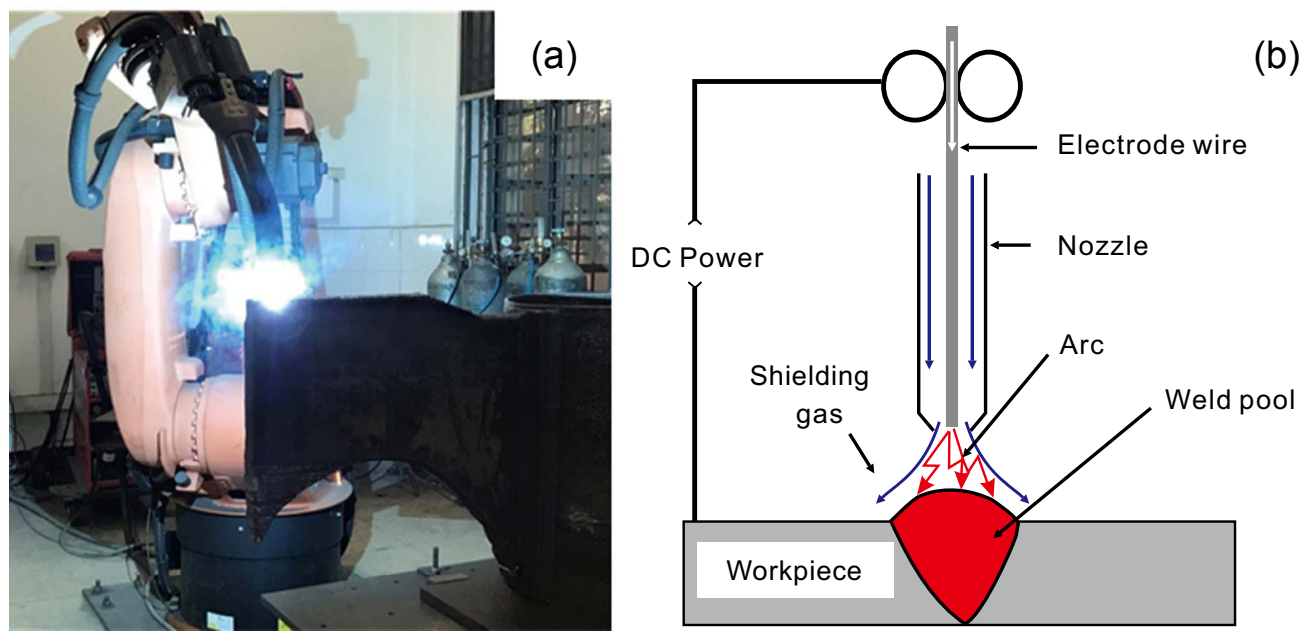

Fig. 11: Equipment (a) and schematic diagram (b) of wire arc additive manufacturing
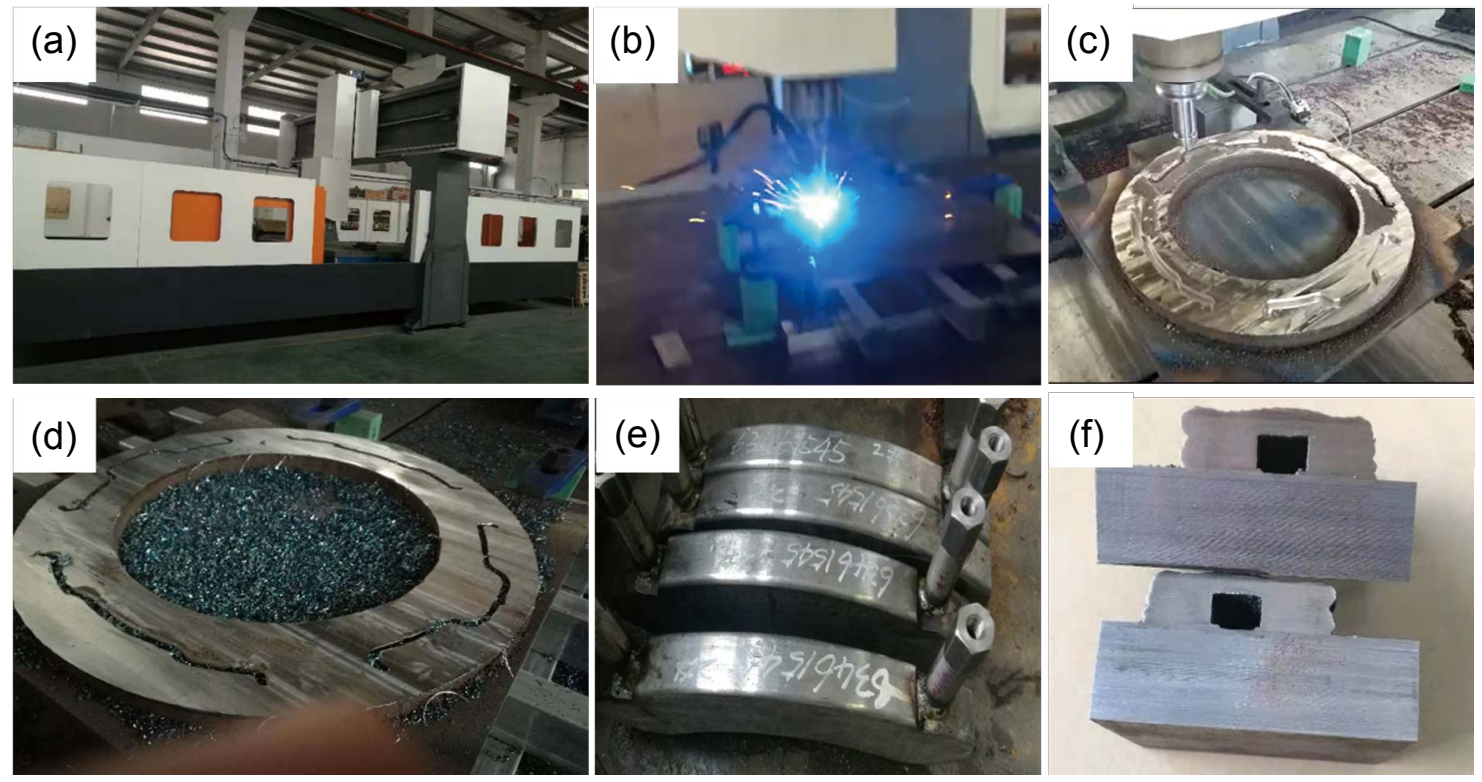

Fig. 12: Wire arc additive/machining subtractive manufacturing technology: (a) forming equipment; (b) wire arc additive manufacturing process; (c) machining subtractive manufacturing process, (d-f) mold with smooth inner runner

\section{Summary}

Focusing on SLS additive manufacturing of complex fusible molds, sand molds (cores) and ceramic molds, research on raw materials-equipment-processes-simulations has been carried out. The method of distilling cooling and uniform temperature reduction was adopted to prepare high-performance powder that meets both the requirements of SLS and casting processes.
A series of SLS additive manufacturing equipment was independently developed, and the process optimization method based on the combination of orderly segmentation of graphics and scanning commutation was proposed, which solved the problems of poor connection strength, easy deformation and low precision of the mold boundary. Finally, the adaptive 
meshing of complex geometric entities was realized, and the numerical simulation software of large and complex castings was independently developed, which increased the calculation efficiency by more than 3 times. Based on the above research results, the SLS integral casting mold was successfully applied to the integral casting of key metal components such as titanium alloy intermediate casing, high-strength stainless steel turbo pump casing and centrifugal wheel, and vermicular iron sixcylinder diesel engine cylinder head. Focusing on the 3DP additive manufacturing of complex sand molds, 3DP printing equipment was developed and equipped with self-developed system software and control units to form sand molds as a whole. Finally, the aspects of additive manufacturing for future casting innovation were introduced, including integrated additive manufacturing of metal core bone-variable zone performance complex sand mold, integrated additive manufacturing of ceramic core and shell, additive/subtractive manufacturing technology of die casting mold with conformal cooling channels.

\section{Acknowledgements}

This study was supported by the Principle and Method of Integrated Laser 3D Printing of Metal Core-Variable Area Performance Complex Sand Mold (Grant No. U1808216), and the Aero Engine and Gas Turbine Major Special Fundamental Research Fund Project (2017-VII-0008-0102).

\section{References}

[1] Shi Y S. The industrial application and industrialization development of 3D printing technology. Machine Design and Manufacturing Engineering, 2016, 45: 11-16.

[2] Wang H M. Material's fundamental issues of laser additive manufacturing for high-performance large metallic components. Acta Aeronautica et Astronautica Sinica, 2014, 35: 2690-2698.

[3] Yang Y R, Li L, Zhao J. Mechanical property modeling of photosensitive liquid resin in stereolithography additive manufacturing: Bridging degree of cure with tensile strength and hardness. Materials \& Design, 2019, 162: 418-428.

[4] Subramanian K, Vail N, Barlow J, et al. Selective laser sintering of alumina with polymer binders. Rapid Prototyping Journal, 1995, 1: 24-35

[5] Wu J M, Chen A N, Liu M Y, et al. Preparation of ceramic materials used for selective laser sintering and related forming methods. Materials China, 2017, 36: 575-582.

[6] Liu K. Investigation on the hybrid technology of laser sintering/ cold isostatic pressing about ceramic powder. Doctoral Dissertation, Huazhong University of Science and Technology, Wuhan, 2014
[7] http://www.huake3d.com, August 21, 2021

[8] Jiang Zilin, Zhou Jianxin, Yin Yajun. A refined mesh generation method for arbitrary three-dimensional geometry of casting. Advance Material Research, 2013, 753-755: 1303-1307.

[9] Mao Y W, Li J W, Wei Q S, et al. Study on the process of threedimensional printing bronze 663. Electromachining \& Mould, 2020, 05: 52-64.

[10] Mostafaei A, Elliott A M, Barnes J E, et al. Binder jet 3D printing -Process parameters, materials, properties, modeling, and challenges. Progress in Materials Science, 2021, 119: 100707.

[11] Upadhyay M, Sivarupan T, El Mansori M. 3D printing for rapid sand casting - A review. Journal of Manufacturing Processes, 2017, 220: 29211.

[12] Le Néel Tugdual A, Mognol P, Hascoët J Y. A review on additive manufacturing of sand molds by binder jetting and selective laser sintering. Rapid Prototyping Journal, 2018, 24(8): 13251336.

[13] Lores A, Azurmendi N, Agote I, et al. A review on recent developments in binder jetting metal additive manufacturing: Materials and process characteristics. Powder Metallurgy, 2019, 62(5): 267-296.

[14] Zhao D, Guo W, Zhang B, et al. 3D sand mould printing: A review and a new approach. Rapid Prototyping Journal, 2018, 24(2): 285-300.

[15] https://www.aniwaa.com/product/3d-printers/voxeljet-vx4000 May 10, 2021.

[16] https://www.aniwaa.com/product/3d-printers/exone-exerial, May 10, 2021.

[17] http://www.fhzl.co, May 10, 2021.

[18] http://www.nanjixiong.com/thread-129161-1-1.html, May 10, 2021.

[19] https://cn.3dsystems.com, May 10, 2021.

[20] http://www.soligentechnologies.com, May 10, 2021.

[21] Yang Q, Lu Z L, Huang F X, et al. Research on status and development trend of laser additive manufacturing. Aeronautical Manufacturing Technology, 2016, 12: 26-31.

[22] Mitra S, de Castro A R, El Mansori M. On the rapid manufacturing process of functional 3D printed sand molds. Journal of Manufacturing Processes, 2019, 42: 202-212.

[23] Hodder K J, Chalaturnyk R J. Bridging additive manufacturing and sand casting: Utilizing foundry sand. Additive Manufacturing, 2019, 28: 649-660.

[24] Liu W W. Research progress on key manufacturing technology of aeroengine blades. Aeronautical Manufacturing Technology, 2016, 21: 50-56.

[25] Maravola M, Conner B, Walker J, et al. Epoxy infiltrated 3D printed ceramics for composite tooling applications. Additive Manufacturing, 2019, 25: 59-63.

[26] Yu S F, Yu R Z, He T Y, et al. Wire arc additive manufacturing and its application: Research progress. Materials China, 2021, 40: 198-209.

[27] Zhou Y. Design and manufacture of impeller conformal cooling mold based on 3D. Plastics Science and Technology, 2017, 3: $76-80$. 